\title{
Infancia y división sexual del trabajo: visibilizando a las niñas trabajadoras en el servicio do- méstico de Perú ${ }^{1}$
}

\author{
Iskra Pávez \\ Universidad Alberto Hurtado \\ ipavez@uahurtado.cl
}

\begin{abstract}
SÍNTESIS
El objetivo de este artículo es reflexionar sobre las experiencias y resistencias de las niñas y las adolescentes que trabajan en el servicio doméstico en Perú, que viven en condiciones de exclusión social y participan en el Movimiento de Niñas, Niños y Adolescentes Trabajadores Organizados de Perú (MNNATSOP). Para ello se consideran los aportes del enfoque del Protagonismo Infantil, la Sociología de la Infancia y la Perspectiva de Género en cuanto a la división sexual del trabajo en la niñez. Se analiza el contexto de origen y las situaciones de migración laboral que viven las niñas, la invisibilización del trabajo doméstico que realizan, el rol del dinero, la discriminación y vulneración de sus derechos, cómo se expresan los roles de género, sus expectativas y las formas en que participan protagónicamente en organizaciones sociales infantiles.
\end{abstract}

\begin{abstract}
This paper reflects on the experience and strengths of girls and adolescents who work as domestic servants in Peru, living in social exclusion and participate in the Movement of Children and Adolescents Organized Workers of Peru (MNNATSOP). This will consider the contributions of prominence Child approach, Sociology of Children and the Gender Perspective as to the sexual division of labour in childhood. We analyse the origin and context of labour migration situations experienced by girls, the invisibility of domestic work, and the role of money, discrimination and violation of their rights, how they express gender roles, expectations and ways involved in social organizations.
\end{abstract}

Palabras clave: Niñas, trabajo infantil, división sexual del trabajo, género, protagonismo infantil.

Key words: Girls, Child Labour, Domestic Work; Gender; Child Agency. 


\section{Introducción}

Por ella nadie apostaba, su futuro se nublaba y no había hecho más que empezar ${ }^{2}$

En la cosmovisión andina, se valora el aporte que realizan las niñas y los niños a sus familias y comunidades mediante las labores domésticas, agrícolas o económicas que realizan, algunas de manera gratuita y otras, de modo pagado. Según Cussiánovich (2002), históricamente la cultura andina ha apreciado el trabajo que realizan las niñas y los niños como un mecanismo de aprendizaje de valores sociales y humanos que se complementan con la educación formal recibida en la escuela y la educación informal de las familias. No obstante, desde fines de los años 70 y debido a una gran crisis económica vivida en Perú -que significó despidos, cierre de industrias y el aumento del desempleo- comenzaron las niñas y los niños a emplearse de manera remunerada como una forma de contribuir económicamente a sus familias. En ese contexto, en el año 1976, surge en Perú el Movimiento de Adolescentes y Niños Trabajadores Hijos de Obreros Cristianos (en adelante MANTHOC). Actualmente, en él participan aproximadamente 2.500 niñas, niños y adolescentes trabajadores (en adelante NATs). En el VI Encuentro Nacional del MANTHOC, realizado el 21 de Marzo de 1996, surge el Movimiento de Niñas, Niños y Adolescentes Trabajadores Organizados de Perú (en adelante MNNATSOP) que aglutina además a otras organizaciones de niñez trabajadora. Actualmente este movimiento congrega alrededor de 14.000 NATs a nivel nacional, cuyo objetivo principal es la promoción de los derechos y la incidencia política a través del protagonismo de las niñas y los niños trabajadores en sus comunidades, escuelas, familias y lugares de trabajo. ${ }^{3}$ Tanto el MANTHOC como el MNNATSOP son apoyados por personas adultas que están organizadas en el Movimiento Nacional de Colaboradores de Niños, Niñas y Adolescentes Trabajadores del Perú (MOVINCOLNATS).

Estos movimientos asumen la "valoración crítica" del trabajo realizado por las niñas, los niños y adolescentes y defienden su 
derecho a trabajar en condiciones adecuadas, que contribuyan al aprendizaje, socialización y desarrollo personal, reflexionando y denunciando la discriminación, marginación y explotación que afecta a NATs de Perú y el mundo entero (Liebel y Martínez, 2009). Estas organizaciones reconocen que las niñas, los niños y adolescentes trabajadores sufren discriminación por su condición de pobreza y además experimentan la exclusión social y política por su condición infantil; por ello elaboran un discurso de incidencia política y crítico con la postura abolicionista del Trabajo Infantil que tienen agencias internacionales como la Organización Internacional de Trabajo (en adelante OIT) y denuncian que las políticas públicas de infancia no contemplan la opinión ni participación de las propias niñas y niños trabajadores en tanto actores sociales. Se cuestiona que se vea a la niñez trabajadora con el estereotipo de víctima recreando la visión del trabajo infantil como un problema social, sin considerar los factores estructurales y económicos, como la pobreza y exclusión social y política en que viven las niñas y los niños trabajadores, es decir, que se les vea como un problema y no como la solución (Figueroa, 2011:18).

El presente artículo surge como fruto de una estancia de investigación postdoctoral realizada por la autora en el año 2011 en el Instituto de Formación para Educadores de Jóvenes-Adolescentes y Niños Trabajadores de América Latina y el Caribe (en adelante IFEJANT) de Lima; a través de esta institución se realizó visitas a las comunidades de Arequipa, Andahuaylas e Iquitos donde se llevó a cabo observación participante de las actividades ejecutadas por las niñas y los niños trabajadores en general, y especialmente con niñas que trabajan en el servicio doméstico y que participan protagónicamente en el MNNATSOP y MANTHOC.

\section{Paradigma del Protagonismo Infantil: reflexionando sobre el derecho de las niñas al trabajo}

De acuerdo a Cussiánovich (2002) y Liebel (2009), la Convención de los Derechos del Niño promulgada por la ONU en 1989, reconoce la dignidad humana de las niñas y los niños desnaturalizando la sumisión o discriminación que sufren por su edad, ya que les comprende como sujetos de derechos. Ciertamente, la propia Conven- 
ción abre la posibilidad de la participación infantil, sin embargo no desde una mirada protagónica, sino mediatizada generalmente por el mundo adulto, ya sea la familia, la escuela o el Estado. Cussiánovich y Figueroa (2009: 87) sostienen que inspirados en el Enfoque de Derechos, el paradigma del protagonismo infantil va más allá porque identifica en la niñez la capacidad de actuar, conocer, cuestionar y transformar su entorno social, político, cultural y económico de manera protagónica desde la propia posición social y generacional que ocupan las niñas y los niños, en compañía del mundo adulto, pero no subordinados a éste. Se trata de una participación protagónica y coprotogónica. Esta cuestión se manifiesta, por ejemplo, identificando los aportes económicos y sociales que realizan las niñas y los niños trabajadores a sus familias, comunidades y a su propio proceso de desarrollo personal y de participación en las organizaciones infantiles. El paradigma del protagonismo se interpreta como un proyecto político emancipador que aspira a reconocer, valorar, respetar y tomar en cuenta a las niñas y los niños trabajadores como actores sociales y protagonistas con capacidad de acción social "presente" tomando distancia crítica de la postura proteccionista o abolicionista del trabajo infantil centrada principalmente en el "futuro". Según Cussiánovich y Figueroa (2009:91), el protagonismo es un horizonte existencial y conceptual desde y hacia el que se encamina la acción y reflexión de las organizaciones infantiles. Se asume que el protagonismo es inherente a la condición humana, por ello se habla de co-protagonismo, porque no es una cuestión individual, sino una condición social. En el caso de la infancia es en clara compañía con el mundo adulto, pero no subordinada a éste.

Al respecto, la declaración de principios del MNNATSOP (citado en Cussiánovich y Figueroa, 2009:91) señala lo siguiente:

Artículo 14: "Consideramos que el protagonismo es un derecho de todo pueblo, de todo ser humano y como tales, los niños como conjunto de infancia gozamos de este derecho y del derecho a ejercerlo".

Artículo 15: "Ser protagonista es para nosotros un responsabilidad que nos exige calidad, excelencia en las iniciativas, en las opiniones, en las propuestas que hagamos para que éstas tengan fuerza social, posibilidades de acogida".

Artículo 16: "Somos protagonistas, pero debemos aprender a 
serlo. El MNNATSOP es un espacio para formarnos una personalidad protagónica, es decir, humilde, con autonomía; flexible, con identidad propia; tolerante, con convicciones fundamentales; emprendedora, con iniciativas sólidas; responsable, con gran imaginación; solidaria en la promoción del protagonismo de los demás".

De la cita anterior se desprende el concepto de "participación protagónica", es decir, que las niñas y los niños tienen el derecho y la capacidad de participar en aquellos asuntos que les conciernen con la posibilidad de generar una real incidencia política en ellos. Se trata de una subjetividad que busca emanciparse de todo aquello que merme su autonomía; la participación protagónica es una forma concreta de reconocer la condición humana de la niñez: "no hay desarrollo de la condición protagónica sin participación, aunque no toda participación deviene en desarrollo y ejercicio del protagonismo" (Cussiánovich y Figueroa, 2009:92) ${ }^{4}$.

En contraste con el predominio de una visión capitalista de la infancia, donde las niñas y los niños ejercen su protagonismo a través del consumo, la participación protagónica como proyecto político constituye un nuevo horizonte ético, que implica el ejercicio de los derechos en el marco de la ciudadanía infantil (Liebel, 2009). No obstante, la dependencia económica que afecta a la niñez moderna agudiza su condición de minoría respecto al poder adulto, puesto que en el sistema capitalista el hecho de obtener y disponer de dinero significa un mecanismo -aunque no el único- de empoderamiento y capacidad de tomar decisiones. Esta situación de desventaja económica, lleva a Oldman (1994:53) a sugerir que las relaciones generacionales entre el colectivo infantil y el adulto puedan leerse como relaciones de clase social, cuyo estatus económico favorable lo tienen las personas adultas. La situación de dependencia económica lleva a la niñez a una subordinación y paternalización permanentes. Debido al discurso escolarizante que recae sobre la niñez moderna, las niñas y los niños no pueden trabajar remuneradamente de forma (Aguirre, 2008; Cordero, 2012), aunque lo deseen como una motivación para tener dinero, en tanto tienen la obligación de asistir a la escuela y no reciben sueldo por el trabajo escolar que realizan en la sociedad del conocimiento (Qvortrup, 1992, 1994). En este marco, las 
niñas y los niños trabajadores representan una amenaza al estereotipo de niñez deseable. Por ello se organizan acciones adultas para combatir el trabajo infantil, argumentando que son explotados laboralmente y que su deber/derecho es estudiar (Cordero, 2012). Según Liebel $(2007,2009)$, en la niñez trabajadora se articulan las categorías de clases sociales y generacionales porque el hecho de que las niñas y los niños puedan ser "productivos" significa tomar parte activa en la vida económica de sus familias y de nuestra sociedad.

\section{Sociología de la Infancia: las niñas como actoras sociales}

La perspectiva teórica de la Sociología de la Infancia comprende a la niñez en un concepto con tres dimensiones, de acuerdo a la clasificación hecha por Gaitán (2006). La primera como categoría sociológica permanente de la estructura social -aunque sus miembros se renueven constantemente- (Qvortrup 1994). La segunda, como una construcción social alejada de una idea esencialista del ser infantil y expresada de diversas formas a través de la historia en cada contexto sociopolítico, y las niña y los niños como como "actores sociales con capacidad de agencia". No obstante, dicha actoría pocas veces es reconocida en la sociedad y en el ámbito académico debido al adultismo o adultocentrismo que les discrimina, esencialmente, por su edad y por la posición de dependencia económica en que se encuentran, característica de la infancia moderna (Jenks 1982; James y Prout 1997). Un claro ejemplo de la construcción social de la infancia es la promulgación de la Convención Internacional de los Derechos del Niño por parte de la ONU en 1989. Ciertamente, la Convención transformó el concepto moderno de infancia promoviendo una cultura más igualitaria y respetuosa de los derechos infantiles, en las políticas públicas, la educación y las familias. No obstante, la Convención también recrea un determinado concepto de infancia y de las relaciones generacionales de poder que se derivan de éste, al otorgar ciertos derechos y negar otros. Por ejemplo, se debate que no se concedan derechos políticos como el voto o se ignoren los derechos sexuales y reproductivos de las y los adolescentes (Gaitán y Liebel, 2011: 29).

El tercer enfoque, denominado relacional de la Sociología de la Infancia, comprende que las niñas y los niños despliegan relaciones 
de poder generacionales y de género con los otros actores, en sus familias y otros espacios sociales. Alanen (1994:36) y Mayall (2002:24) proponen aprender del género -entendido como un concepto relacional-, para leer a la niñez como un proceso relacional. Esta idea parte de la premisa teórica de que tanto el sistema de género como el generacional, operan paralela y complementariamente, y que ambos organizan las relaciones de las niñas, los niños y las personas adultas en función de las jerarquías de edad y sexo. Esto iimplica asumir la dimensión de poder de género y generacional en el sistema patriarcal (Mayall, 2002:24). Por lo tanto, la deconstrucción de los estereotipos de género también ayuda a deconstruir las imágenes y las representaciones sociales de la niñez, ya que mujeres, niñas y niños (en tanto grupos sociales), están en posición de minoría y subordinación en la cultura patriarcal (Alanen, 1994; Mayall, 2002).

Las relaciones patriarcales tienen múltiples dimensiones de poder. Por un lado, el orden patriarcal significa una jerarquía entre los géneros, con especial dominio de la figura masculina del patriarca sobre la figura femenina. También implica relaciones de subordinación entre las edades: el patriarca tiene autoridad sobre las niñas y los niños, dada su posición generacional y de género. Las relaciones de poder de clase también son ejercidas por el patriarca en el seno de familias o comunidades que gozan de una buena posición económica, ya que el patriarca se yergue como el jefe de familia frente a las personas que realizan las labores despreciadas, como la limpieza y servidumbre (Izquierdo, 1998).

Si bien los niños experimentan las desventajas propias de este sistema cultural, -en tanto están en una posición generacional de subordinación-, una vez que crecen y tienen más edad, van adquiriendo mayor poder respecto a la jerarquía generacional y de género. En cuanto a las niñas, éstas experimentan una doble subordinación generacional y de género, cuando ellas van creciendo adquieren mayores cuotas de poder generacional, pero continúan subordinadas desde el punto de vista de género, incluso como mujeres adultas. (Mayall, 2002).

Desde un análisis feminista, se cuestiona que la biología de los cuerpos de las mujeres sea un destino universal, porque más bien se trata de una cuestión política e histórica. ${ }^{5}$ Alanen (1994:34) sugiere que la Sociología de la Infancia analice aquella representación social 
de la biología infantil que muestra a las niñas y los niños como seres dependientes, carentes de cuidado y control permanente de parte de una "mujer" que desarrolle su maternidad de modo intensivo y abnegado, idealmente en el marco de una familia patriarcal, heterosexual y burguesa. Se trata de la ideología familiar surgida en Europa y Estados Unidos durante la época fordista, caracterizada por el binomio del hombre-ganapán (bread-winner) y la mujer-cuidadora-ama de casa (house-wife) (Benería, 1981, 2005, 2007; Moore, 1991; Molyneux, 1994:144; Izquierdo, 1992; Parella, 2003).

No obstante lo anterior, los intereses de la niñez y de las mujeres no siempre han coincidido en los movimientos feministas o de liberación infantil. Por ejemplo, según Alanen (1994), la teoría feminista no hace propia la liberación infantil, ni siquiera en el caso de las niñas -en tanto sujetos femeninos- porque mantiene una visión adultocéntrica de la niñez, basada en visiones psicológicas/biológicas y, por lo tanto, de inferioridad y subordinación. Aunque Mayall (2002:12) reconoce que ciertas autoras (como Firestone, 1973) ya habían identificado una opresión común entre las mujeres y la niñez, sería preciso que las teorías feministas y los paradigmas de infancia se articulen, para que de esa forma se visibilicen las desigualdades de género y generacionales en ambos grupos sociales.

Si bien existe convergencia epistemológica entre la deconstrucción del sujeto femenino (gracias a la Teoría Feminista) y la desnaturalización del sujeto infantil (gracias a los aportes de la Sociología de la Infancia), en este análisis se considera a la niñez como un grupo homogéneo que sólo tiene pertenencia generacional. Aunque se trata de un aporte valioso, queda pendiente el desafío de visibilizar políticamente la situación de las niñas situadas en múltiples jerarquías de poder, tales como el género, la edad, el territorio, la clase social o el origen étnico o nacional. Develar la situación específica de las niñas en términos generacionales y de género puede ser leído como una cuestión política clave en la investigación feminista y en el estudio sociológico de la niñez, entendida como un grupo social con relaciones de poder y desigualdad (Alanen, 1994; Mayall, 2000, 2002).

En ese sentido, los "girls studies" o "estudios de las niñas" constituyen un acercamiento, desde múltiples dimensiones, hacia la vida particular de las niñas (Lipking, 2009). Se estudian tanto los procesos de socialización -que inciden en su presente y su futuro, cuando 
sean mujeres adultas- $y$ de modo particular se analiza ese espacio socialmente construido y reservado para que lo habiten las niñas cuando están en la posición generacional de la infancia y en el espacio de género, en tanto, mujeres. Si bien dicho espacio está socialmente construido, las niñas lo reproducen y eventualmente lo subvierten, dada las múltiples maneras de vivir sus infancias en cada contexto histórico y territorial. En este marco, el trabajo reproductivo que realizan las niñas resulta un aspecto clave para examinar las desigualdades que experimentan por su condición de género.

\section{Perspectiva de género: las niñas como cuidadoras en la división sexual del trabajo ${ }^{6}$}

La división sexual del trabajo se entiende como la asignación diferenciada de roles, espacios y actividades a las mujeres y los hombres; implica una división jerárquica y binaria del trabajo, separando la producción de la reproducción (Benería y Sen, 1981; Benería, 2005; Molyneux; 1994:147). Este concepto generalmente se aplica en el estudio de los roles que desarrollan las personas adultas, no obstante, los ámbitos donde trabajan las niñas y los niños también reproducen dicha división de género. En este sentido, el estudio de Leyra (2008), sobre las condiciones de trabajo de las niñas en los mercados de Ciudad de México, demuestra que cuando las niñas trabajan remuneradamente realizan labores asociadas a la reproducción social. Así, los niños trabajan en labores asociadas al mundo público, como venta ambulante, labores de carga y descarga, lustrabotas, etc.; mientras, las niñas, en labores típicamente femeninas, como asistentes en tareas de cuidado y en el servicio doméstico. Por su parte, la investigación de Aguirre (2008) sobre las niñas y los niños empacadores también refleja la división sexual del trabajo en la infancia trabajadora. Mientras que el estudio de Vizcarra y Marín (2006), demuestra que el trabajo doméstico gratuito que realizan las niñas y los niños en sus familias -incluso en contextos de comunidades indígenas como es el caso de este estudio- también sigue patrones de género diferenciados, en la medida que ellas y ellos contribuyen al bienestar familiar al realizar tareas de limpieza, cuidado y autocuidado. Sin embargo, tal como plantea Mayall (2002) generalmente no se les reconoce este aporte. 
A pesar que la división sexual del trabajo se basa en algunas diferencias biológicas entre los cuerpos de las mujeres y los hombres, se trata principalmente de un ordenamiento que se fundamenta en construcciones sociales e históricas, y sobre todo económicas y políticas vigentes en nuestras sociedades patriarcales (Moore, 1991; Vizcarra y Marín, 2006). Si bien es innegable la necesidad de que las mujeres participen en la reproducción biológica de los seres humanos (al menos en la gestación y el parto), ${ }^{7}$ la reproducción social como tal, es decir las tareas asociadas a la crianza y el cuidado de los bebés, es una responsabilidad que ha sido asignada social e históricamente a las mujeres, mediante los procesos de socialización de género diferenciados (Moore, 1991; Lamas, 1996).

A partir de la modernidad, el reparto de labores "productivas" y "reproductivas" organiza los espacios donde se llevan a cabo dichas labores: un espacio público para la producción, y uno privado para la reproducción. Para Benería (1981, 2005:85), las tareas asociadas a la reproducción son enseñadas a las niñas como algo "natural", propio de su género, pero transmitidas mediante sutiles mecanismos de poder, control social, y en muchos casos, de manera obligatoria mediante el uso de la violencia. Como se piensa que son tareas "naturales" de las niñas y mujeres, se las persuada para que las hagan "por amor" y sin esperar retribución económica alguna. Por las mismas razones, dichas actividades tienen un escaso prestigio, a la vez que están desvalorizadas económicamente en nuestras sociedades (Borderías y Carrasco, 1994; Molyneux, 1994:122; Hartman, 1994:292).

En el ámbito familiar se enseña y vigila que las niñas, las jóvenes y las mujeres aprendan y realicen este tipo de trabajos a través de los procesos de socialización de género. De esta manera, el hogar y el ámbito privado se convierten en el foco de trabajo para ellas, puesto que es allí donde se desarrollan las actividades domésticas y de cuidado asignadas socialmente, mientras que por el otro lado, se espera que niños y hombres dominen las tareas asociadas al espacio público (Moore, 1991; Vizcarra y Marín, 2006). Sin embargo, la posición económica de clase social y la ubicación geográfica (urbana o rural) de cada familia son factores que inciden en la forma de distribución del trabajo reproductivo entre niñas, niños y mujeres adultas del grupo familiar; ya que si existen los medios económicos suficientes, se contratarán los servicios de otra mujer (niña, joven, pobre, indígena 
o inmigrante) para que realice las labores de limpieza o cuidado de sus hogares y así, se librarán de esta pesada carga (Hartman, 1994).

La división sexual del trabajo implica subordinación de las niñas y mujeres al espacio doméstico como centro de su quehacer y realización personal (house wife), un espacio de la vida privada que, por lo mismo, está altamente invisibilizado. Por otra parte, este sistema dual prepara y exige a los niños y hombres que se desarrollen en el ámbito público y en actividades productivas asociadas a su género, puesto que se les inculca que ellos serán los responsable de mantener económicamente el hogar (bread winner) (Lamas, 1996, Benería, 2005; Saú, 2006) ${ }^{8}$. Lo anterior se sintetiza en la Tabla № 1 (ver anexos).

A pesar que en las sociedades latinoamericanas las mujeres se han incorporado al trabajo remunerado, en los procesos de socialización infantil (juegos infantiles, medios de comunicación, currículo escolar) se sigue transmitiendo la idea de la familia patriarcal, donde las niñas y mujeres están en el espacio privado (siendo las responsables del trabajo doméstico y de cuidado), y los niños y hombres trabajando en el ámbito público (Vizcarra y Marín, 2006). Además, normalmente los trabajos a los que acceden las niñas, jóvenes y mujeres en el mercado laboral, son una extensión del rol doméstico/ cuidador y rápidamente se convierten en nichos altamente feminizados y desvalorizados. Esto demuestra cómo el sistema económico converge con el sistema patriarcal de género, ya que el mercado del trabajo está altamente segregado en función del género y de los roles socialmente atribuidos a niñas y niños, mujeres y hombres adultos (Hartmann, 1994:253, Parella, 2003; Benería, 2005). ${ }^{9}$

El estudio de Soraya Hoyos (2000: 122) revela las graves vulneraciones a sus derechos que experimentan las niñas trabajadoras en sus lugares de trabajo, situación que está altamente invisibilizada. Para esta autora la llamada "feminización de la pobreza" en América Latina afecta en mayor medida a las niñas, los niños y las mujeres adultas, e incide en que las niñas tengan que emplearse fuera del hogar, pero también en que ellas se vean obligadas a asumir más responsabilidades domésticas que sus hermanos de la misma edad, al interior de sus familias. Lo anterior es claramente una expresión de la reproducción de la división sexual del trabajo en la niñez. Por su parte, UNICEF $(1991,2007)$ ha declarado su preocupación e interés de modo particular en la situación de las niñas, porque ellas expe- 
rimentan mayores desigualdades que los niños, mayores niveles de violencia (específicamente sexual), mayor índice de desnutrición, en definitiva mayores vulneración de sus derechos.

En síntesis, el enfoque de género aplicado al estudio de la niñez nos permite analizar los mecanismos y las formas en que las niñas, los niños, las mujeres y los hombres participan diferenciadamente en la división sexual del trabajo y las consecuencias sociales, económicas y políticas que se derivan de ello.

\section{Contextos y experiencias de las niñas trabajadoras en el servicio doméstico en Perú. Análisis de resultados}

Según estimaciones de la propia OIT, el $83 \%$ de quienes realizan labores de trabajo doméstico serían niñas y mujeres. Además, de acuerdo al trabajo de campo desarrollado en IFEJANT, las niñas, las adolescentes y las jóvenes que trabajan en el servicio doméstico tienen bajos niveles de estudio, las condiciones laborales son precarias porque no tienen contrato y los sueldos son muy bajos. Habitualmente el trabajo doméstico se caracteriza por una tendencia hacia la explotación y la vulneración de los derechos laborales, dado que ocurre en el ámbito privado. Debido a su posición de género y edad, las niñas y las adolescentes están más expuestas, que sus pares varones, a sufrir situaciones de violencia psicológica, física y sexual. La mayoría de las niñas que trabajan en el servicio doméstico compagina esta actividad con sus estudios en la jornada alterna a su empleo.

Un estudio realizado por Elvira Figueroa (2011:18), a niñas y niños trabajadores de Perú analiza el aporte económico de éstos al presupuesto familiar. Considerando que el sueldo mínimo en Perú es de 600 soles (224U\$) y la Canasta Básica Familiar asciende a 1.037 soles (400U\$), en un contexto donde el mayor gasto se destina a alimentos; se constató que el sueldo promedio de las niñas y los niños trabajadores en Perú es de 257 soles (96 U\$), lo que representaría un aporte del $35 \%$ a la Canasta Básica Familiar. Sin embargo, en la práctica este estudio (Figueroa, 2011) identificó que el aporte real que realizan las niñas y los niños al presupuesto familiar mensual es de 182 soles (18\%). En otras palabras, del sueldo promedio que obtienen las niñas y los niños trabajadores destinan una gran cantidad de soles al presupuesto familiar y el resto de sus ingresos lo gastan 
en cosas personales, materiales escolares, ropa, golosinas, ahorrar o invertir

Por otro lado, el estudio de Figueroa (2011:18) revela las razones que las niñas y los niños declaran para trabajar, en orden de importancia, son: Ayudar a mi familia (40,2\% de las respuestas); Necesidades escolares (17,5\%); Necesidades personales $(14,7 \%)$; Salir adelante $(10,9 \%)$; Ropa (9,2\%); Ahorrar (5,5\%); Golosinas (1,3\%) y Negocio propio (0,8\%). Como puede observarse los datos reflejan que las necesidades familiares representan una de las mayores motivaciones para trabajar remuneradamente, aunque muy seguido encontramos carencias de índole más individual y vinculadas al crecimiento personal de las niñas y los niños. Por último, este mismo estudio (Figueroa, 2011) muestra que el promedio de horas diarias de trabajo de las niñas y los niños trabajadores en Perú, en general varía entre 4 a 6 horas, lo que nos permite interpretar que compaginan sus estudios con las actividades laborales remuneradas, en la Tabla № 2 se pueden ver los detalles (ver anexos).

En el siguiente apartado se presenta el análisis de la información recopilada en la observación participante, mediante las categorías utilizadas en el trabajo de campo: contexto y migración laboral; invisibilización; el rol del dinero; la discriminación; la vulneración de derechos; los roles de género; las expectativas y el protagonismo de las niñas trabajadoras.

\subsection{Contexto y migración laboral de las niñas trabajadoras}

En Andahuaylas -comunidad de la sierra, ubicada en Los Andes peruanos-, las familias más pobres y excluidas, generalmente provienen de comunidades rurales e indígenas quechua-hablantes, no poseen los medios ni las oportunidades para ofrecer una manutención mínima o los estudios básicos a sus hijas. En estos casos, se contactan con otras familias que viven en ciudades y tienen mejores condiciones de vida, estas familias realizan una especie de "amadrinamiento o apadrinamiento" de las niñas prometiéndoles educación a cambio de que colaboren con las labores domésticas de sus hogares, trabajando en modalidad llamada de "cama adentro" (internas). Esta "migración laboral" infantil resulta un "alivio" económico para las familias con más necesidades económicas, pero con un alto costo emocional y humano particularmente para las niñas. Si bien 
históricamente la migración campo-ciudad ha sido utilizada como una estrategia de supervivencia, especialmente entre las familias pobres e indígenas, durante la década de 1980, por el conflicto político vivido en Perú, esta situación se agravó: muchas familias indígenas de la zona andina vieron en la migración de sus hijas e hijos la única forma de que ellas y ellos accedieran a una mejor calidad de vida.

Por su parte, en Arequipa -comunidad de la costa-sierra, ubicada al sur de Lima- existe el Programa de Servicio Doméstico de la Congregación Religiosa María Inmaculada; que atiende aproximadamente a 100 niñas de 8 a 14 años de edad. Al igual que ocurre en Andahuaylas, aquí las niñas provienen de comunidades rurales y/o indígenas tales como la Sierra de Arequipa, Puno y Ayacucho. La casa de la Congregación ofrece alojamiento a las niñas a cambio de que ellas no abandonen sus estudios y aporten económicamente a la mantención de la casa con el dinero que ganan trabajando en el servicio doméstico.

\subsection{Invisibilización de las niñas trabajadoras}

Al igual que el caso que investiga Aguirre (2008), en el Programa de Arequipa, recién mencionado, se realiza una especie de capacitación a las niñas sobre las labores domésticas (limpieza, cocina, cuidado infantil, etc.), de manera tal que puedan cumplir con las demandas que les realizan las familias empleadoras cuando ya están trabajando. Estas familias consideran el trabajo doméstico que realizan las niñas solo como una pequeña ayuda en sus hogares. Sin embargo, el nivel de carga laboral resulta excesivo para las capacidades de las niñas y las adolescentes. De todas las labores domésticas encomendadas, el cuidado de otras niñas y niños resulta lo menos tedioso, porque en esos momentos ellas pueden descansar y jugar.

Por su parte, en la ciudad de Andahuaylas, se estima que la mayoría de las viviendas tienen niñas trabajando en el servicio doméstico; incluso, las propias autoridades locales así como profesionales de los servicios sociales, de salud o educación lo hacen como una forma de "ayudar" a las familias más pobres. Cuando el MANTHOC ha intentado realizar censos para cuantificar este fenómeno, las familias empleadoras lo niegan, argumentando que se trata de "la ahijada o sobrina que viene del campo", además, a las niñas se les exige que traten a sus patronas como "tías o madrinas". Por lo tanto, el trabajo 
doméstico que realizan las niñas queda doblemente invisibilizado, por la familia empleadora y por ellas mismas.

De acuerdo al concepto de división sexual del trabajo de Benería (2007) y Leyra (2008), se trata de modelos de reproducción de género en la infancia, porque las labores que realizan las niñas son invisibilizadas y negadas por las familias, pero se ven directamente beneficiadas de ese trabajo. El estudio de Aguirre (2008) encuentra similares resultados sobre esta forma de interpretar el trabajo doméstico que realizan las niñas y los niños como una "ayuda", cuestión que invisibiliza su aporte.

\subsection{El rol del dinero}

Las niñas que trabajan en el servicio doméstico, tanto en modalidad externa como interna obtienen un sueldo mensual promedio de 200 Soles Peruanos (76 U\$ aproximadamente). Como ya se ha dicho, el trabajo que realizan las niñas en el servicio doméstico es un tipo de gestión doméstica de los hogares para los cuales trabajan e incluye actividades de limpieza, cocina, compras familiares y cuidado infantil de otras niñas, niños y bebés. El estudio de Vizcarra y Marín (2006) halla similares actividades asignadas a las niñas en función de la división sexual del trabajo.

Las niñas que vienen de comunidades pobres, rurales e indígenas se comprometen a enviar dinero, es decir, remesas económicas, para ayudar a la manutención de sus familias. Debido a que en ocasiones la contratación de las niñas resulta como fruto de un acuerdo entre las personas adultas de los grupos familiares (de origen y empleador), no se les da el dinero directamente a ellas, sino a sus madres o padres y ellas sólo reciben pequeñas "propinas diarias". En los casos donde la contratación de la niña trabajadora fue acordada como un "amadrinamiento o apadrinamiento", la familia empleadora no paga con dinero los servicios prestados por la niña, ni a ella ni a su familia; ya que solo existe el compromiso de brindar manutención y estudios a cambio de la "pequeña" ayuda o compañía que ella realiza. Nuevamente, se identifica la invisibilización del aporte al trabajo de reproducción social que realizan las niñas, al igual que el estudio de Aguirre (2008).

Cuando las niñas tienen la oportunidad de negociar el sueldo y las condiciones laborales con las familias empleadoras, logran mayores 
cuotas de poder y libertad, dentro del marco de acción minoritario que tienen para desenvolverse, tal como dice Mayall (2002). No obstante, las niñas que no reciben dinero por el trabajo doméstico que realizan pierden la posibilidad de negociar sus propias condiciones laborales y tampoco pueden gestionar diariamente sus gastos. Estos argumentos son similares a los planteados en los estudios de niñez trabajadora en Perú de IFEJANT (Liebel, 2009) o los hallazgos encontrados en los estudios de niñas trabajadoras en los mercados de ciudad de México de Leyra (2008) o en las tiendas de Tiuana de Aguirre (2008).

De acuerdo a Leyra (2008) y Benería (2005), en el contexto capitalista actual, el hecho de no recibir dinero por el trabajo doméstico que realizan, las niñas se ven situadas en múltiples posiciones de vulnerabilidad y exclusión que facilitan escenarios de explotación llevadas a cabo en el ámbito privado, y por lo tanto, de difícil intervención y análisis público. Tomando distancia crítica de una imagen que refuerza la idea miserabilista de la niñez trabajadora, se trata de situaciones cercanas a la "neoesclavitud", porque no se reconoce el aporte económico que ellas realizan.

\subsection{Discriminación}

En el trabajo de campo se constató que las niñas que provienen de comunidades indígenas y/o rurales sufren la discriminación y el racismo tanto en sus lugares de trabajo, como en sus escuelas. Algunas niñas son discriminadas por razones étnicas o por prejuicios racistas porque visten sus trajes típicos o hablan en quechua, su idioma natal. Además, como ya se ha mostrado, las niñas que trabajan en el servicio doméstico habitualmente provienen de familias que viven en situación de pobreza y exclusión, lo que aumenta las probabilidades de sufrir discriminaciones por esta condición de clase social en la que viven.

\subsection{Vulneración de derechos}

De acuerdo a Leyra (2009) y Benería (2005), el hecho que el trabajo reproductivo se lleve a cabo en el ámbito privado propicia situaciones de vulnerabilidad para quienes lo llevan a cabo. Además, la posición generacional y de género de las niñas trabajadoras en el servicio doméstico las sitúa en posiciones de mayor vulnerabilidad 
frente a sus pares varones, por ejemplo. De este modo, las niñas son construidas socialmente como "objetos" de protección o de abuso, pero no como "sujetos de derecho". Dada la vulnerabilidad socialmente construida en torno a las niñas en general, en el caso específico estudiado sobre las niñas trabajadoras en Perú, ellas son trabajadoras experimentan situaciones de maltrato psicológico, físico y abuso sexual de parte de hombres jóvenes y adultos que son sus empleadores. Las niñas que trabajan en modalidad interna, es decir, que viven en las casas donde trabajan, están más expuestas a sufrir este tipo de vulneraciones a diferencia de quienes no viven en sus lugares de trabajo. Las consecuencias del maltrato infantil en ámbitos laborales, no solo afecta la autoestima y la confianza de las niñas, sino que además representan una grave vulneración a sus derechos y en ocasiones aumentan su sensación de soledad en circunstancias en que están alejadas de sus redes familiares o de amistades debido a las largas jornadas laborales y escolares.

\subsection{Roles de género}

En Andahuaylas, y siguiendo las categorías de Leyra (2009), la división sexual en la niñez trabajadora se plasma en que los niños trabajan en la venta de periódicos y lustrabotas; mientras, las niñas lo hacen en el sector de hostelería, comercio y servicio doméstico. Los niños que trabajan como lustrabotas tienen como esperanza lograr algún tipo de movilidad social hacia empleos con mayor estatus, por ejemplo, como conductores en el transporte local. A su vez, las niñas que trabajan en el servicio doméstico en modalidad de internas aspiran a obtener un empleo en el mismo rubro, pero como externas y de este modo, lograr movilidad social. Cuando los niños trabajan en el servicio doméstico, como ayudantes de cocina o como cargador de las compras familiares, ellos lo ocultan porque sienten vergüenza. En estos casos, se invisibiliza la condición de niño trabajador por razones de género, ya que es un trabajo asignado socialmente a las niñas y las mujeres, que tiene cierto desprestigio, por lo tanto, se debe ocultar de posibles burlas e interpretaciones normativas.

Por otro lado, en la distribución de las tareas al interior de los grupos de niñez trabajadora que participan en MANTHOC y MNNATSOP, generalmente las niñas desarrollan labores asociadas a la reproducción social, como la preparación de alimentos o la limpieza 
de los lugares que utilizan; mientras, los niños asumen prácticas en el espacio público, como realizar juegos u ocupar los puestos de liderazgo en los espacios comunitarios de base. Evidentemente, la reproducción de la división sexual del trabajo, de modo particular en la infancia, incide en la ocupación de posiciones de poder que dejan en desventaja a las niñas, porque ven vulnerados sus derechos a jugar y descansar, o a ejercitar prácticas de dominio de la palabra y resolución de conflictos en las posiciones de liderazgo al interior de los grupos infantiles. Como una manera de responder a esta debilidad, tanto MANTHOC como MNNATSOP exigen que la representación a nivel regional y nacional sea compartida entre una niña y un niño.

\subsection{Expectativas de las niñas trabajadoras}

En Arequipa, cuando las niñas cumplen los 14 años deben abandonar la casa religiosa, en ese momento las alternativas que ellas tienen son varias; en el mejor de los casos, tras acabar sus estudios obtienen titulaciones técnicas y, en menor medida, ingresan a estudios universitarios. Pero, generalmente sus opciones se reducen a volver a sus hogares de origen; emplearse como trabajadoras domésticas externas; emigrar hacia otras ciudades; y algunas comienzan a convivir con sus parejas y tienen un embarazo precoz.

En Andahuaylas, una vez que las niñas, las adolescentes y las jóvenes están insertas en el empleo doméstico tienen la expectativa de migrar a ciudades turísticas o con mayores oportunidades laborales como Ica, Nazca o Lima, con la esperanza de encontrar mejores condiciones laborales, aunque en el mismo rubro del servicio doméstico. De hecho, en los grupos sociales de niñas trabajadoras, se cuentan los relatos y las experiencias de otras jóvenes que ya han emigrado hacia esas ciudades, y sobre las oportunidades y desafíos que han debido enfrentar, entre los cuales se resaltan las situaciones de discriminación por provenir de la sierra peruana.

\subsection{Protagonismo de las niñas trabajadoras}

Las niñas y las adolescentes trabajadoras en el servicio doméstico participan protagónicamente en organizaciones infantiles del MNNATSOP. Particularmente, en Andahuaylas existe una organización de niñas trabajadoras ligada al MANTHOC, quienes participan en diversas actividades de autogestión. Estas niñas tienen un grupo or- 
ganizado que desarrolla actividades deportivas con otros grupos de niñas, niños y jóvenes de la provincia. Además, al interior del propio grupo se eligen sus representantes, en este caso una niña cumple el rol de Delegada ante el Comité Provincial, quien también tiene una representación regional donde se encuentra con otras representantes a nivel nacional. El trabajo de la Delegada consiste en coordinar las actividades, visibilizar la situación de las niñas trabajadoras del servicio doméstico y denunciar condiciones de explotación, porque es una realidad bastante desconocida, invisible e invisibilizada, tanto para la sociedad peruana en general, como al interior del propio movimiento de NATs.

En relación a este punto, un mecanismo frecuentemente utilizado por las niñas trabajadoras del servicio doméstico son las nuevas tecnologías, de entre las cuales destacan las redes sociales, utilizadas para mantenerse en contacto y coordinación con otras niñas y niños trabajadores, tanto a nivel local, nacional y transnacional en el marco de su participación en los Movimientos de NATs.

\section{Conclusiones}

Las pautas culturales y sociales reconstruyen relaciones de desigualdad de género en la niñez, las cuales obstaculizan el ejercicio de los derechos de las niñas. A su vez, se recrean los dispositivos de la división sexual del trabajo, donde niñas y adolescentes generalmente realizan labores asociadas a la reproducción social, altamente estigmatizadas, invisibilizadas y con escaso valor tanto social como económico.

La articulación de los supuestos teóricos del protagonismo infantil, la sociología de la infancia y la perspectiva de género logran mostrar las desigualdades de género y generacionales que vive la niñez en general, y las niñas en particular. En las páginas precedentes hemos podido comprender la visibilización política de la situación específica de las niñas, como actoras sociales situadas en múltiples jerarquías de género, edad, territorio, de clase social y origen étnico o nacional.

La división sexual del trabajo implica una organización jerárquica y binaria del trabajo que separa la producción de la reproducción. La división sexual del trabajo en la niñez se expresa en que los niños 
trabajan en labores asociadas al mundo público (venta ambulante, carga y descarga, etc.), mientras que las niñas se emplean en labores típicamente femeninas, como asistentes en tareas de cuidado y el servicio doméstico. Los trabajos a los que acceden las niñas representan una extensión del rol doméstico y cuidador que se ha socializado, se trata de labores altamente feminizadas y desvalorizadas y debido a su posición de género y edad, las niñas y las adolescentes están más expuestas, que sus pares varones, a sufrir situaciones de violencia psicológica, física y sexual.

A lo largo de estas páginas hemos podido comprobar que la situación de las niñas trabajadoras del hogar es bastante desconocida, invisible e invisibilizada, tanto para la sociedad peruana en general, como al interior del propio movimiento de NATs. Se trata de una niñez que enfrenta las paradojas de la modernidad, en tanto experimenta graves exclusiones socioeconómicas y territoriales, sumadas a la mencionada discriminación. Sin embargo, al mismo tiempo participan de una generación infantil global, la cual desarrolla agencias, culturas y espacios de identidad a través de nuevas formas, un tanto desconocidas para el mundo adulto.

\section{Anexos}

Tabla № 1. La división sexual del trabajo

\begin{tabular}{|c|c|}
\hline Ámbito privado/doméstico & Ámbito público \\
\hline $\begin{array}{c}\text { Trabajo asignado a las niñas y } \\
\text { mujeres }\end{array}$ & $\begin{array}{c}\text { Trabajo asignado a los niños y } \\
\text { hombres }\end{array}$ \\
\hline Asociados a la reproducción social & $\begin{array}{c}\text { Asociados a la producción } \\
\text { económica }\end{array}$ \\
\hline $\begin{array}{l}\text { Se consideran naturalmente } \\
\text { femeninos }\end{array}$ & $\begin{array}{l}\text { Se consideran naturalmente } \\
\text { masculinos }\end{array}$ \\
\hline $\begin{array}{c}\text { Escasa valoración social y } \\
\text { económica }\end{array}$ & Alta valoración social y económica \\
\hline \multicolumn{2}{|l|}{ Indispensable para la vida humana } \\
\hline $\begin{array}{l}\text { Invisible e invisibilizado: no se } \\
\text { considera "trabajo" }\end{array}$ & $\begin{array}{c}\text { Visible y visibilizado: puede llegar } \\
\text { a ser explotación }\end{array}$ \\
\hline $\begin{array}{l}\text { Implica labores de socialización, } \\
\text { cuidado, servicio doméstico, } \\
\text { limpieza. }\end{array}$ & $\begin{array}{l}\text { Mercado laboral primario y } \\
\text { terciario }\end{array}$ \\
\hline
\end{tabular}

Fuente: elaboración propia a partir de Benería $(1981,2005,2007)$ y 


\section{Tabla № 2. Promedio de horas diarias de trabajo de NATs}

\begin{tabular}{|l|l|l|}
\hline $\begin{array}{c}\text { Número de horas diarias } \\
\text { trabajadas }\end{array}$ & No de niñas y niños & \% del total \\
\hline 4 a 6 horas & 199 & $51,8 \%$ \\
\hline 1 a 3 horas & 72 & $18,8 \%$ \\
\hline 6 a 8 horas & 58 & $15,1 \%$ \\
\hline 8 a 10 horas & 31 & $8,1 \%$ \\
\hline + 10 horas & 24 & $6,3 \%$ \\
\hline Total & $\mathbf{3 8 4}$ & $\mathbf{1 0 0} \%$ \\
\hline
\end{tabular}

Fuente: Figueroa, 2011:18.

\section{Notas}

1. Una versión anterior del presente artículo fue el Informe Final de la estancia de investigación postdoctoral realizada por la autora en el año 2011 en el Instituto de Formación de Educadores de Jóvenes, Adolescentes y Niños Trabajadores de Perú (IFEJANT). Agradezco de modo particular a Manfred Liebel (Alemania), Elvira Figueroa, Alejandro Cussiánovich (Lima), Rolando Willems (Andahuaylas), Gisella Correa (Iquitos), Tabita Lozano, Pilar Cristóbal, Carmen Velásquez (Lima), por toda su acogida, colaboración y apertura. También quiero agradecer a Jasmira Suri Quispe, delegada nacional de MNNATSOP, y Miguel Valenzuela, delegado nacional de MANTHOC.

2. La Niña, Mala Rodríguez.

3. A su vez, el MNNATSOP forma parte del Movimiento Latinoamericano y del Caribe de Niños, Niñas y Adolescentes Trabajadores (MOLACNATS). Este último se coordina transnacionalmente con el Movimiento Africano de Niños y Jóvenes Trabajadores (MAEJT) y el Movimiento de niños/as y adolescentes trabajadores de Asia Central y Sur.

4. En el proceso educativo se promueve a través de la utilización de una filosofía y metodología denominada Pedagogía de la Ternura (Cussiánovich, 2002).

5 "Keeping children out of sight in feminist research helps to keep even a number of women's problems invisible (...). It has therefore kept women and children lumped together and left them sharing the same oppression" (Alanen, 1994:34).

6. Una versión preliminar de este apartado fue publicada en Pavez, 2011.

7. Firestone (1973) sugiere un feminismo utópico que altere las formas de reproducción de la especie como las conocemos hasta ahora, con el objetivo de que las mujeres que realmente deseen embarazarse lo hagan, que no sea un mandato u obligación moral, ni menos el destino natural de todas las mujeres.

8. Tal y como señala Vandelac (1994:184), el mercado de trabajo se basa en la visión de un trabajador masculino, es decir, encarnado en un hombre adulto visto como un ser egoísta, racional y competitivo que se desenvuelve bien en el ámbito laboral 
y en el mundo público. Esto es lo que se conoce como homo oeconomicus, un ser que prescinde de los ámbitos de la reproducción social, como si se hicieran por simple obra de un milagro económico.

9. Esta segmentación se visualiza tanto horizontal, en el sentido de asignar diferentes tipos de ocupaciones a hombres y mujeres, como verticalmente, manifestándose en la entrega de menores salarios, menor prestigio social y escasas posibilidades de ascenso, desencadenando menores cuotas de poder para las mujeres $\mathrm{y}$, por lo tanto, para las ocupaciones a las que ellas acceden. Muchos empleos femeninos son informales, se caracterizan por inestabilidad temporal, deficientes condiciones laborales y en general escasa protección social. Por último, otro rasgo de esta segmentación se constata en que las mujeres continúan concentrándose mayoritariamente en el sector de los servicios, en contra posición a los hombres, que ocupan puestos de dirección o como obreros en la industria (Harman, 1994; Parella, 2003). Por otro lado, también es preciso considerar la gran variedad de trabajo informal que realizan las mujeres más pobres desde sus hogares y como una forma de contribuir al presupuesto familiar. Este tipo de subempleo o economía sumergida en ocasiones puede ser para una gran cadena multinacional que se beneficia de los intermediarios y desarrolla la producción a muy bajo coste (Benería, 2005:122).

\section{Bibliografía}

Aguirre, Lina. Intervención institucional y aprendizaje de las niñas y niños empacadores en las tiendas de autoservicio de Tijuana, México. Convergencia, vol. 15, núm. 48, Univesidad Autónoma del Estado de México, 71-102, 2008.

Alanen, Leena. "Gender and Generation: Feminism and the Child Question'. Qvortrup, Jens et al (Eds.) (1994). Childhood Matters: Social Theory, Practice and Politics. Viena: Avebury-European Centre Vienna. Volume 14, 27-41, 1994.

Benería, Lourdes. Género, desarrollo y globalización. Por una ciencia económica para todas las personas. Barcelona: Editorial Hacer, 2005.

Benería, Lourdes. “Trabajo productivo/reproductivo, pobreza, y políticas de conciliación en América Latina: consideraciones teóricas y prácticas". En: Astelarra, Judith. (Coord.). Género y cohesión social. Madrid: Fundación Carolina, 107-120, 2007.

Benería, Lourdes y Sen, Gita. “Accumulation, reproduction and women's role in economic development, Boserup Revisited", Signs, 7(2), 279-298, 1981.

Borderías, Cristina, Carrasco, Cristina y Alemany, Carme (Comp.). Las mujeres y el trabajo. Rupturas conceptuales. Barcelona: Icaria-Fuhem, 1994.

Borderías, Cristina y Carrasco, Cristina. "Introducción. Las mujeres y el trabajo: aproximaciones históricas, sociológicas y económicas". En: Borderías, Cristina et al. (Comp.) Las mujeres y el trabajo. Rupturas conceptuales. Barcelona: Icaria-Fuhem, 18-92, 1994.

Cussiánovich, Alejandro. Aprender la condición humana. Ensayo sobre pedagogía de la ternura. 2a edición. Lima: IFEJANT, 2010.

Cussiánovich, Alejandro y Figueroa, Elvira. “Participación protagónica: ¿Ideología 
o cambio de paradigma?". En: Liebel, Manfred y Martínez, Marta. (Eds.). Infancia y derechos humanos: hacia una ciudadanía participante y protagónica. Lima: IFEJANT, 83-99, 2009.

Figueroa, Elvira. . Revista Internacional de NATS, 2011.

Firestone, Shulamith. La dialéctica del sexo. En defensa de la revolución feminista. Barcelona: Kairós. 1976[1973].

Hartmann, Heidi. “Capitalismo, patriarcado y segregación de los empleos por sexos". En: Borderías, Cristina et al. (Comp.) Las mujeres y el trabajo. Rupturas conceptuales. Barcelona: Icaria-Fuhem, 253-294, 1994.

Hoyos, Soraya. “¿Y quién la mandó a ser niña?”: El trabajo infantil doméstico desde una perspectiva de género. En: Acosta, Gladys; García Méndez, Emilio y Hoyos, Soraya (Eds). Trabajo Infantil Doméstico: ¿Y quién la mandó a ser niña?. Bogotá: UNICEF-Tercer Mundo Editores, 113 - 123, 2000.

Izquierdo, María Jesús. El malestar de la desigualdad. Madrid: Cátedra, 1998.

Lamas, Marta. "La perspectiva de género", La Tarea, Revista de Educación y Cultura, 8, 1996.

Leyra, Begoña. Trabajo infantil femenino: niñas trabajadoras en Ciudad de México. Tesis Doctoral, Departamento de Antropología Social, Madrid: Universidad Complutense de Madrid, 2009.

Liebel, Manfred. Entre protección y emancipación. Derechos de la infancia y políticas sociales. Madrid: Experto en Políticas Sociales de Infancia-Universidad Complutense de Madrid, 2007.

Liebel, Manfred. “Niños ciudadanos. Hacia una política de infancia desde los niños como titulares activos de derecho". Ponencia presentada en I Jornades de Polítiques d'Infància a Catalunya, Generalitat de Catalunya, Barcelona, España, 20 de noviembre de 2008. Inédito.

Liebel, Manfred. "Sobre la historia de los Derechos de la infancia". En: Liebel, Manfred y Martínez, Marta. (Eds.). Infancia y derechos humanos: hacia una ciudadanía participante y protagónica. Lima: IFEJANT, 23-40, 2009.

Liebel, Manfred y Martínez, Marta. (Eds.). Infancia y derechos humanos: hacia una ciudadanía participante y protagónica. Lima: IFEJANT. 2009.

Lipkin, Elline. Girls' studies. Berkley: Seal Press, 2009.

Mayall, Berry. "Towards a Sociology for Childhood. Thinking from children's lives". Glasgow: Open University Press-McGraw-Hill Education, 2009 [2002].

Molyneux, Maxine. "Más allá del debate sobre el trabajo doméstico". En: Borderías, Cristina et al. (Comp.) Las mujeres y el trabajo. Rupturas conceptuales. Barcelona: Icaria-Fuhem, 111-150, 1994.

Moore, Henrietta. Antropología y feminismo. Madrid: Ediciones Cátedra, 2009 [1991].

Parella, Sònia. Mujer, inmigrante y trabajadora. La triple discriminación. Barcelona: Anthropos, 2003.

Pavez Soto, Iskra. "Migración infantil: rupturas generacionales y de género. Las niñas peruanas en Barcelona y Santiago de Chile". Tesis Doctoral. Departamento de Sociología, Universitat Autònoma de Barcelona, 2011.

Saú, Victoria. "Reproducción social de las identidades de género: de niñas a mujeres". En: Rincón, Ana. (Cord.). Congreso Internacional SARE 2005: "Niñas son, mujeres serán". Bilbao: Emakunde/Instituto Vasco de la Mujer, 16-20, 2006. 
UNICEF. Convención Internacional de los Derechos del Niño. Ginebra: ONU, 1989.

UNICEF. "La niña. Una inversión para el futuro". Sección para el desarrollo del Programa para la Mujer. New Cork: United Nations Children's Fund, 1991.

UNICEF. “Estado Mundial de la Infancia. La mujer y la infancia: El doble dividendo de la igualdad de género". Nueva York: UNICEF, 2007.

Vandelac, Louise. "La economía doméstica a la salsa mercantil... o las valoraciones monetarias del trabajo doméstico". En: Borderías, Cristina et al. (Comp.) Las mujeres y el trabajo. Rupturas conceptuales. Barcelona: Icaria-Fuhem, 151208, 1994.

Vizcarra, Ivonne y Marín, Nadia. "Las niñas a la casa y los niños a La Milpa: la construcción social de la infancia mazahua". Convergencia, vol. 13, núm. 40, Universidad Autónoma del Estado de México, 39-67, 2006. 